\title{
Sphingolipid metabolism products: potential new players in the pathogenesis of bortezomib-induced neuropathic pain
}

\author{
Albert Alé ${ }^{1}$, Andreas A. Argyriou ${ }^{2}$, Jordi Bruna ${ }^{1,3}$ \\ ${ }^{1}$ Institute of Neurosciences and Department of Cell Biology, Physiology and Immunology, Universitat Autònoma de Barcelona, Centro de Investigación \\ Biomédica en Red sobre Enfermedades Neurodegenerativas (CIBERNED), Bellaterra, Spain; ${ }^{2}$ Department of Neurology, Saint Andrew's State General \\ Hospital of Patras, Patras, Greece; ${ }^{3}$ Unit of Neuro-Oncology, Hospital Universitari de Bellvitge-ICO l'Hospitalet, IDIBELL, l'Hospitalet de Llobregat, \\ Barcelona, Spain \\ Correspondence to: Jordi Bruna, MD, PhD. Unit of Neuro-Oncology, Hospital Universitari de Bellvitge-ICO L'Hospitalet, 08907, Barcelona, Spain. \\ Email: 35078jbe@comb.cat. \\ Comment on: Stockstill K, Doyle TM, Yan X, et al. Dysregulation of sphingolipid metabolism contributes to bortezomib-induced neuropathic pain. J \\ Exp Med 2018;215:1301-13.
}

Submitted Oct 12, 2018. Accepted for publication Oct 22, 2018.

doi: $10.21037 /$ atm.2018.10.53

View this article at: http://dx.doi.org/10.21037/atm.2018.10.53

Chemotherapy-induced peripheral neurotoxicity (CIPN) is one of the major dose-limiting adverse events of widely used drugs in both the oncologic and hematologic setting (1). Among its cardinal symptoms, neuropathic pain is frequently present (2). In particular, the incidence of bortezomib-induced peripheral neurotoxicity (BIPN) and neuropathic pain ranges from $14-45 \%$ and $5-39 \%$, respectively, in myeloma multiple patients. BIPN is more frequently developed in pretreated patients, compared to those being chemotherapy-naïve $(3,4)$, and this difference mostly accounts for the wide variability in the observed incidence rates. Bortezomib is the first proteasome inhibitor introduced in clinical practice. The mechanisms underlying the pathogenesis of peripheral neurotoxicity in bortezomibtreated patients are, yet, not fully elucidated $(3,4)$.

We read with great interest the recently published paper by Stockstill et al. (5), which sought to shed further light on the pathogenetic mechanisms of bortezomibassociated neuropathic pain. The scientific background of the latter study was based on the increase of sphingolipid ceramide biosynthesis as pro-apoptotic signaling in cancer cells, together with the evidence that ceramide and other sphingolipid metabolites, specifically the sphingosine-1phosphate (S1P), are potent molecules with inflammatory and nociceptive actions. On that context, the authors demonstrated in preclinical in vivo models that bortezomib increased the S1P and ceramide levels, among others sphingolipid metabolism products, in dorsal horn spinal cord (DHSC) astrocytes. These astrocytes are activated by SP1 receptor 1 (SP1R), leading to neuroinflammation through the overexpression of proinflammatory cytokines and over-release of the neurotransmitter glutamate in DHSC neurons, as fundamental mechanisms to generate the bortezomib-induced neuropathic pain. After showing increased levels of the main sphingolipid products in DHSC astrocytes as reaction to bortezomib exposure, two different approaches were applied to demonstrate the involvement of sphingolipid dysregulation in pain and prevent this adverse event of bortezomib administration. First, at a pharmacological level, authors used the S1PR1 antagonists FTY720 (fingolimod) and NIBR14, which prevented the animals from developing mechanical allodynia and hyperalgesia. Second, from a loss-of-function perspective, they also used knockdown mutants of SPR1 expression in astrocyte [driven by glial fibrillary acidic protein (GFAP)] and siRNA for SPR1, and in line with the previous models, showed a delayed development of neuropathic pain compared with the control group.

Moreover, researchers observed a parallel hyper-reactivity of DHSC astrocytes located in the lamina I and II and proinflammatory cytokines tumour necrosis factor (TNF) and interleukin-1 $\beta$ (IL-1 $\beta$ ), whereas a decrease of antiinflammatory cytokines IL-1 and IL-4 levels was also noted. In addition, the increased labeling of GFAP and 
high pro-inflammatory cytokine levels in the DHSC were reverted by the administration of the S1PR1 antagonist FTY720 (fingolimod). As result of this observation, the authors suggest a feed-forward loop between inflammatory cytokines and sphingolipid dysregulation on the basis of the ability of TNF and IL-1 $\beta$ to activate the major enzymes involved in the biosynthesis of ceramide and S1P pathways, according to previously published evidence from other groups (6). To fully explore the ability of SP1 in modulating the function of nervous system pain pathways, they also recorded the excitatory postsynaptic currents from neurons, which receive input from the primary afferents in the spinal outer lamina II.

The analysis of the experimental results showed an increase in both the frequency and amplitude of postsynaptic currents, in bortezomib-treated group. Furthermore, the samples from animals (rats) co-treated with the S1PR1 antagonist demonstrated a decreased frequency of synaptic currents, while the inverse effect (increase) was noted with the administration of an S1PR1 agonist. The interpretation of these observations thoroughly supports the implication of bortezomib in the alterations of glutamatergic signaling in DHSC neurons, driven by the S1PR1 activation at the presynaptic level.

Finally, the authors suggest that the relationship between the pro-inflammatory cytokine state and the sphingolipid dysregulation, together with the increase in the glutamatergic signaling may promote, reinforce and/or maintain the neuronal sensitization in the central nervous system (CNS). Moreover, they propose as potential useful treatment approach the use of S1PR1 antagonist drugs, which are already available in clinical practice to treat other CNS disorders.

Despite the interesting and relevant findings reported by Stockstill and collaborators (5), several points need clarification and contextualization. Generally, there is a debate and controversy among the researchers in the field regarding which are the appropriate animal models to use for testing CIPN. Some investigators use treatment schedules consisting of short course and low doses of drug administration. These models develop neuropathic pain features that are related with the cytostatic administration, as in the Stockstill et al. paper, and are used to explore the CIPN pathogenesis and new treatments strategies to alleviate the neuropathic component of pain. Noteworthy, neuropathological and/or nerve conduction impairments are required to establish the diagnosis of peripheral neuropathy. However, it is uncertain if these treatment schedules are capable to experimentally induce CIPN with features clinically resembling it in cancer patients. In fact, the single bortezomib doses used by the authors were low, even if chemotherapy was administrated during a more prolonged time, compared to the single dose used in other animal models in which impairments in behavioral and nerve conduction studies were evident, and also nerve and dorsal root ganglia histopathological abnormalities were reported $(7,8)$. Taken into account that the cumulated dose is the main risk factor to develop CIPN in chemotherapy treated patients (1), it sounds rational that the models with more extended schedules and higher single dose drug administration, thus reaching total equivalent cumulated doses similar to the patients, are the most appropriate to characterize and investigate the pathogenesis of CIPN through evidence of neuropathological and neurophysiological abnormalities. In addition, another point in the experimental procedure that needs clarification is whether the researchers were blinded to treatments and randomization when conducting behavioral tests, as this in our view consist a crucial issue in any animal behavioral assessment.

On the other hand, it is intriguing why the authors have not extended their research on sphingolipid dysregulation and on the effects of the SP1R blockade in Schwann and satellite cells, or even in dorsal root neurons, especially when it is well known that bortezomib is not able cross the blood brain barrier, and its main neurotoxic effects are in the peripheral nervous system, both in patients and animal models $(3,4,6,7)$. Although bortezomib cannot penetrate into the CNS, the authors link the dysregulation on sphyngolipid metabolism of DHSC astrocytes with a peripheral increase in pro-inflammatory cytokines induced by the bortezomib exposure. In support of their assumption, it is previously demonstrated in an experimental model of chronic bortezomib exposure, a predominant increase in TNF and IL-6 expression on dorsal root ganglia neurons, mainly in the small size neurons, compared to spinal cord and peripheral nerve. Moreover, it was also evident that drugs blocking the TNF action, also partially prevent the neurotoxicity induced by bortezomib (9).

It is well known that bortezomib-induced peripheral nerve damage can be characterized as a pure sensory neuronopathy $(3,4,6,7)$. However, if the pro-inflammatory environment is responsible of the DHSC astrocytes changes, it still remains to be explained why these inflammatory cytokines have not evoked relevant changes in motor or sensory neurons, which are also exposed to 
this inflammatory environment. Likewise, further research is warranted to elucidate why the enhanced release of glutamate from presynaptic vesicles by S1P; the second contributing factor to neuropathic pain genesis according to the authors' results, have not produced collateral effects on motor neurons, especially over the small and vulnerable glutamate motor neurons (10).

Another significant question that arises from the paper by Stockstill et al. (5) relates with the specificity of the sphingolipid dysregulation mechanism as a contributing factor for generating bortezomib-induced neuropathic pain. Interestingly, the same research group has previously involved the same dysregulated pathway in the pathogenesis of paclitaxel-induced neuropathic pain (11). To clarify this point, it is advisable to further test the effect of carfilzomib over the DHSC astrocyte sphingolipid metabolism wields, as this drug is a second-generation proteasome inhibitor without exerting relevant neurotoxic adverse events (12).

The last but not least questionable point upon the Stockstill et al. (5) publication concerns the assumed recommendation to treat patients with FTY720 (fingolimod) which is already available in clinical practice, to treat CNS disorders, such as multiple sclerosis. Patients with multiple myeloma or other hematologic malignancies are at high risk to be immunocompromised. Therefore, a drug impairing the ability of cytotoxic CD8 T cells to destroy their target cells, thus further compromising the immunity (13), may not be the best approach for alleviating the neuropathic pain component in these patients.

Nevertheless, in our opinion the study by Stockstill et al. (5) is of significance, because it is presenting for the first-time experimental results to support the significance of sphingolipid dysregulation in the pathogenesis of bortezomib-induced neuropathic pain, thus potentially shedding further light in the pathogenetic mechanism of BIPN as a whole. Thus far, the main mechanisms involved in the BIPN are related with the cytoskeletal alterations caused by an increased microtubule stabilization due to $\alpha$-tubulin polymerization, mitochondrial dysfunction, dysregulation of neurotrophins, and neuroinflammation processes mediated by neuronal expression of IL- 6 and tumor necrosis factor $(3,4)$. The role of sphingolipid dysregulation can indeed play a potential role in the painful phenotype of BIPN, as Stockstill and collaborators have demonstrated. However, to reinforce this potential involvement, similar results have to be replicated in appropriate chronic BIPN models, and the assessment should ideally have to be extended to the peripheral nervous cellular compartment (Schwann and satellite cells, and sensory dorsal root ganglia neurons).

Finally, it is clinically important to take into account several particular points to contextualize the BIPN research. CIPN in bortezomib-treated patients possess distinctive and intriguing characteristics compared with other forms of CIPN. First, this adverse effect might not be a class effect, as second-generation proteasome inhibitors, such as carfilzomib, are less neurotoxic than bortezomib (14), while being equally or more effective in treating multiple myeloma. Second, improvements in symptoms severity and a relatively quick resolution of BIPN occur after chemotherapy cessation in most patients and animal models with bortezomib-related CIPN. This reversibility of symptoms soon after chemotherapy discontinuation is contrary with the persistence of symptoms and notably slow neurological recovery that is observed in patients with CIPN related to other neurotoxic drugs, particularly platinums and taxanes (1). Last, the BIPN is more frequent in patients with multiple myeloma than in those with other cancers. This observation supports the possibility that multiple myeloma itself plays a part in the development of BIPN $(15,16)$. As such, it should be emphasized that only fully considering the clinical phenotype of this toxic neuropathy, and accordingly, with the adequate adjust of the preclinical research settings, we would allow to shed further light on the pathogenesis of bortezomib-induced peripheral nerve damage with or without comprising the component of neuropathic pain.

\section{Acknowledgements}

None.

\section{Footnote}

Conflicts of Interest: The authors have no conflicts of interest to declare.

\section{References}

1. Argyriou AA, Bruna J, Marmiroli P, et al. Chemotherapyinduced peripheral neurotoxicity (CIPN): an update. Crit Rev Oncol Hematol 2012;82:51-77.

2. Geber C, Breimhorst M, Burbach B, et al. Pain in chemotherapy-induced neuropathy--more than neuropathic? Pain 2013;154:2877-87.

3. Alé A, Bruna J, Navarro X, et al. Neurotoxicity induced 
by antineoplastic proteasome inhibitors. Neurotoxicology 2014;43:28-35.

4. Argyriou AA, Cavaletti G, Bruna J, et al. Bortezomibinduced peripheral neurotoxicity: an update. Arch Toxicol 2014;88:1669-79.

5. Stockstill K, Doyle TM, Yan X, et al. Dysregulation of sphingolipid metabolism contributes to bortezomibinduced neuropathic pain. J Exp Med 2018;215:1301-13.

6. Liang Y, Ma S, Zhang Y, et al. IL-1 $\beta$ and TLR4 signaling are involved in the aggravated murine acute graft-versushost disease caused by delayed bortezomib administration. J Immunol 2014;192:1277-85.

7. Bruna J, Udina E, Alé A, et al. Neurophysiological, histological and immunohistochemical characterization of bortezomib-induced neuropathy in mice. Exp Neurol 2010;223:599-608.

8. Carozzi VA, Renn CL, Bardini M, et al. Bortezomibinduced painful peripheral neuropathy: an electrophysiological, behavioral, morphological and mechanistic study in the mouse. PLoS One 2013;8:e72995.

9. Alé A, Bruna J, Morell M, et al. Treatment with antiTNF alpha protects against the neuropathy induced by the proteasome inhibitor bortezomib in a mouse model. Exp Neurol 2014;253:165-73.

10. Mancuso R, Navarro X. Amyotrophic lateral sclerosis: Current perspectives from basic research to the clinic.

Cite this article as: Alé A, Argyriou AA, Bruna J. Sphingolipid metabolism products: potential new players in the pathogenesis of bortezomib-induced neuropathic pain. Ann Transl Med 2018;6(Suppl 1):S78. doi: 10.21037/atm.2018.10.53
Prog Neurobiol 2015;133:1-26

11. Janes K, Little JW, Li C, et al. The development and maintenance of paclitaxel-induced neuropathic pain require activation of the sphingosine 1-phosphate receptor subtype 1. J Biol Chem 2014;289:21082-97.

12. Siegel D, Martin T, Nooka A, et al. Integrated safety profile of single-agent carfilzomib: experience from 526 patients enrolled in 4 phase II clinical studies. Haematologica 2013;98:1753-61.

13. Ntranos A, Hall O, Robinson DP, et al. FTY720 impairs CD8 T-cell function independently of the sphingosine-1phosphate pathway. J Neuroimmunol 2014;270:13-21.

14. Dimopoulos MA, Sonneveld P, Siegel D, et al. Carfilzomib and pomalidomide in patients with relapsed and/or refractory multiple myeloma with baseline risk factors. Ann Oncol 2015;26:2247-56.

15. Coiffier B, Osmanov EA, Hong X, et al. Bortezomib plus rituximab versus rituximab alone in patients with relapsed, rituximab-naive or rituximab-sensitive, follicular lymphoma: a randomised phase 3 trial. Lancet Oncol 2011;12:773-84.

16. Richardson PG, Xie W, Mitsiades C, et al. Single-agent bortezomib in previously untreated multiple myeloma: efficacy, characterization of peripheral neuropathy, and molecular correlations with response and neuropathy. J Clin Oncol 2009;27:3518-25. 